\title{
Diagnostic dilemmas and results of treatment for chronic constipation
}

\author{
C W Keuzenkamp-Jansen, C J Fijnvandraat, C M F Kneepkens, A C Douwes
}

\begin{abstract}
Chronic functional constipation (CFC) may be difficult to recognise and information regarding its long term prognosis is scarce. The records of 244 children with CFC, aged 0-18 years, were analysed for symptoms at presentation and results of treatment, and long term outcome was evaluated by means of a telephone interview in 137 patients discharged for more than one year. The patients presented with a great variety of symptoms, only $22 \%$ having infrequent defecation of increased consistency, another $22 \%$ having an obviously normal defecation pattern. The mean duration of treatment was 13 months. At the time of discharge, $69 \%$ of the patients still used laxatives. At a median of four years after discharge, $66 \%$ of the children were free of symptoms and without medication, $39 \%$ having experienced a recurrence. It is concluded that CFC may be difficult to recognise and can be alleviated by an intensive laxative regimen. Recurrence of symptoms is common, but the long term prognosis is good in most patients.

(Arch Dis Child 1996;75:36-41)
\end{abstract}

Keywords: chronic constipation, plain abdominal radiography, laxatives.

Although constipation in children is frequently encountered by general practitioners as well as paediatricians and psychologists, its diagnosis and treatment remain difficult and its prognosis uncertain..$^{1-4}$ After exclusion of such relatively rare anatomical causes of constipation such as Hirschsprung's disease, anal atresia and spinal disease, and secondary causes such as being bedridden and hypothyroidism, in most cases chronic functional constipation (CFC) can be diagnosed. CFC is easily diagnosed, when decreased frequency of defecation and increased size and consistency of the faeces are the main symptoms. The diagnosis can be missed in many cases, ous other explanation should be available, and they should subside after colonic evacuation.

The treatment of CFC requires at least several months to be successful. Evacuation of the colon followed by maintenance laxation, toilet training, and parental counselling and support are important aspects of the treatment. Even with optimal treatment, relapses frequently occur, both during treatment and afterwards. Until now, only one systematic survey of the presenting complaints of CFC has been reported, and little is known of the long term outcome. ${ }^{5}$ The purpose of this study was, therefore, to identify the presenting symptoms of CFC in childhood and to evaluate its long term outcome.

\section{Patients and methods}

PATIENTS

A total of 244 children with CFC were included out of a total of 409 children referred to our paediatric gastroenterological outpatient department, between 1976 and 1991, for complaints that were eventually shown to be caused by constipation. Roughly half of the patients were referred by family practitioners and half by paediatricians. Patients were included when they fulfilled the following criteria: signs and symptoms consistent with CFC; intake plain abdominal radiography available (in children older than 1 year) ${ }^{6}$; no other cause for constipation found as judged by selective screening; no history of anal surgery; and a treatment period at our outpatient department of at least six weeks. A total of 165 children were not eligible for the following reasons (numbers in parentheses): referred for diagnosis of Hirschsprung's disease (65), history of psychomotor retardation (7), history of anal dilatation (10), seen only for diagnostic purposes (30), less than three visits (25), no laxative treatment instituted (9), and records incomplete (19).

\section{DIAGNOSIS AND TREATMENT REGIMEN}

All children were initially evaluated and treated by two of the authors (ACD and CMFK). Medical histories were obtained and a complete physical examination was performed. In all children a plain abdominal radiograph was made and evaluated using the Barr score. ${ }^{6}$ In our experience, this score is useful when applied to children over 1 year of age.

Once CFC was diagnosed, the children were treated according to the standard three stage therapy schedule used at our centre. In the first stage, the evacuation phase, the colon is evacuated employing a stool softener (lactulose or lactitol) in combination with a mild irritant (bisacodyl or sodium picosulphate, depending \footnotetext{
however, if only these symptoms are relied on. The symptoms of CFC, including the altered defecation characteristics, are primarily caused by the delayed passage of faeces leading to colonic faecal retention; this implies that other symptoms, such as recurrent abdominal pain, may be prominent. For this reason we prefer to define CFC as a condition in which chronic faecal retention, not caused by an anatomical abnormality, induces abdominal and/or systemic symptoms. For these symptoms no obviAC Douwes

\author{
Correspondence and reprint \\ requests to: Dr Douwes. \\ requests to: Dr Douwes. \\ Department of
Paediatrics, Free
University Hospital, \\ PO Box 7057, 1007
Netherlands \\ CW Keuzenkamp-Jansen \\ CJ Fijnvandraat
CMF Kneepkens \\ University Hospital,
PO Box 7057, 1007 MB
}


on the age of the child). Mineral oil is added when the bulk of faecal retention is located in the distal colon and rectum and especially in the presence of scybala. Rectal medication (phosphate enemas) is used only in the first few days of treatment in case of huge faecal impaction in the rectosigmoid, and in case of difficult to treat recurrences, as an adjunct to oral medication. In the evacuation phase, the laxative doses are high enough to produce semiliquid stools, in order to obtain evacuation of the colon and to prevent active withholding of defecation. Parents and children receive oral and written information on the nature of the condition and the rationale for our therapeutic regimen. When old enough the children are told to visit the toilet twice daily and take enough time for defecation. No dietary advice is given at this stage apart from the introduction of wholemeal bread, if necessary. Initially, weekly support is offered, either by means of outpatient visits or by telephone. The aim of the second stage is to prevent colonic reaccumulation of faeces. The laxative dose is decreased until the child produces soft stools daily, and tapered at intervals of two to three weeks until the lowest effective dose is reached. Finally, in the third stage, a fibre enriched diet is introduced and laxatives are gradually stopped. Insufficient progress or relapses prompt intensification of treatment. This may include a one to two week hospital admission in order to obtain evacuation in a clinical setting.

Dependent on the clinical judgment of the attending consultant and the willingness of patient and parents, a child psychologist may be consulted. Referral usually takes place when one of the following factors is present: constipation or symptoms refractory to medical treatment without obvious organic explanation; overt psychosocial pathology judged to interfere with medical treatment; or parents asking for help in the guidance of their constipated child.

\section{DATA COLLECTION}

The information used in this study was extracted from the patient records using a structured data collection form. When the child had been discharged for more than one year from the outpatient department by 1 June 1991, the parents were approached for a telephone interview. They were questioned about any relapses and any continuing use of laxatives. They were considered to be cured when they reported no actual complaints attributable to CFC and did not use any laxatives in the last six months.

STATISTICAL ANALYSIS

Frequency distributions were calculated for symptoms and treatment variables. The follow up data were analysed by constructing a Kaplan-Meier curve of the persistence of complaints for three age groups. Differences between these groups were tested with a log rank test (Mantel-Cox). Associations between outcome at follow up and variables about history or treatment variables were tested with the $\chi^{2}$ test.

\section{Results}

PATIENT CHARACTERISTICS AND SYMPTOMS AT PRESENTATION

The charts of 135 boys and 109 girls were reviewed (male to female ratio 1.23:1). The ages of the children at the onset of symptoms and the total delay between onset of symptoms and start of laxative treatment are depicted in fig 1 . The long delays were principally caused by patient's delay, that is, the time between the onset of complaints and the first medical consultation. For 154 patients both the patient's delay and the doctor's delay were known. In this group medians and first and third quartiles for the patient's delay were $0.7,2.4$, and 9.0 months; for the doctor's delay, $0,0.8$, and 5.1 months; and for total delay, 1.6, 5.4, and 17.0 months, respectively. The longest patient's delay as reported by the parents was more than 16 years. There was no relation between age at start of symptoms and total delay.

The reasons for presentation at the outpatient department, as well as the symptoms mentioned during history taking, are shown in table 1. 'Constipation' as such (that is, hard, infrequent stools) was the presenting symptom in not more than $31 \%$ of the children. Encopresis, defined as involuntary loss of stools including soiling, was found in half of the children over 3.5 years of age, and in $40 \%$ of them was the principal reason for referral (table 1). As judged by a plain abdominal radiograph, constipation was present in all of them and they were treated not differently from the whole group. Interestingly, 15 children $(6 \%)$ presented as an emergency because of acute abdominal pain. Most children (60\%) experienced at least two other symptoms in addition to their main reason for referral (table 1). Information on defecation frequency and consistency, available on 210 children $(86 \%)$ is given in table 2 .
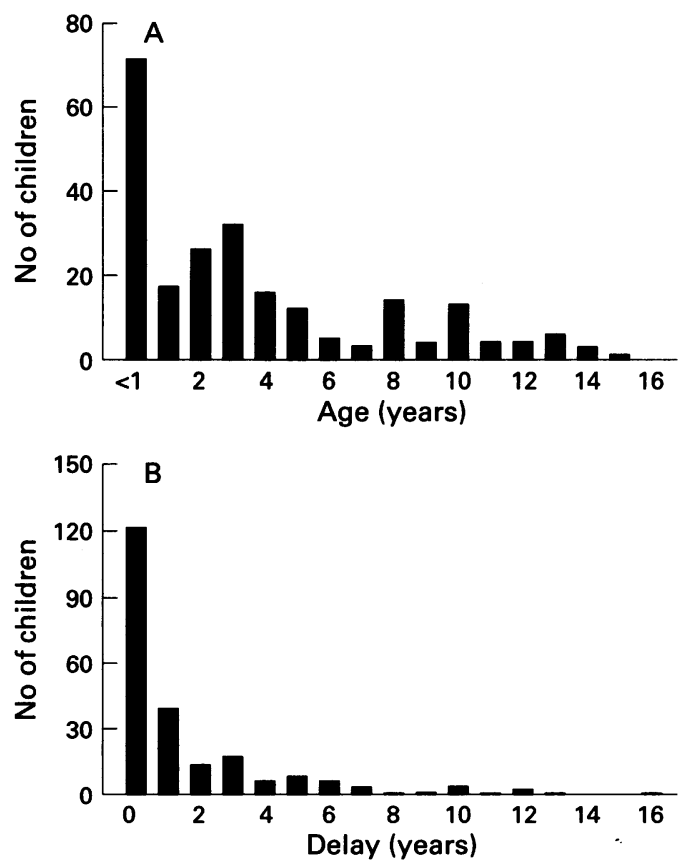

Figure 1 (A) Age at start of treatment $(n=231)$ and $(B)$ total delay before laxative treatment started $(n=226)$; median age was 3 years and median delay 10 months. 
Table 1 Reasons for presentation and symptoms of the 244 children; figures are number (\%)

\begin{tabular}{lcc}
\hline Symptoms & $\begin{array}{l}\text { Reasons for } \\
\text { presentation }\end{array}$ & $\begin{array}{l}\text { Symptoms at } \\
\text { time of } \\
\text { presentation * }\end{array}$ \\
\hline Abdominal pain & $77(31.6)$ & $135(55.3)$ \\
Constipation & $75(30.7)$ & $106(43.4)$ \\
Encopresis, soiling & $34(13.9)$ & $87(49.7)^{\dagger}$ \\
Anorexia & $1(0.4)$ & $69(28.3)$ \\
Malaise & $4(1.6)$ & $38(15.6)$ \\
Painful defecation & $2(0.8)$ & $36(14.8)$ \\
Diarrhoea & $18(7.4)$ & $34(13.9)$ \\
Enuresis & $1(0.4)$ & $26(10.7)$ \\
Vomiting & $5(2.0)$ & $24(9.8)$ \\
Rectal bleeding & $2(0.8)$ & $18(7.4)$ \\
Headache & 0 & $17(7.0)$ \\
Fear of defecation & $3(1.2)$ & $15(6.1)$ \\
Nausea & 0 & $15(6.1)$ \\
Low grade fever & 0 & $14(5.7)$ \\
Distended abdomen & 0 & $11(4.5)$ \\
Perianal problems & $1(0.4)$ & $10(4.1)$ \\
Flatulence & 0 & $10(4.1)$ \\
Sleeping problems & 0 & $9(3.7)$ \\
Urinary tract infections & $1(0.4)$ & $9(3.7)$ \\
Delayed faecal continence & $10(4.1)$ & 0 \\
Food allergy & $6(2.5)$ & 0 \\
Other & $4(1.6)$ & $14(5.7)$ \\
* &
\end{tabular}

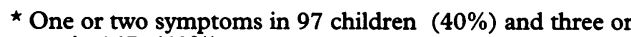
more in $147(60 \%)$

+ Calculated as a percentage of the 175 children over 3.5 years of age, the age at which continence may be expected.

Table 2 Defecation patterns of 210 children at time of presentation; figures are number (\%)

\begin{tabular}{|c|c|c|c|c|}
\hline \multirow[b]{2}{*}{ Consistency } & \multicolumn{4}{|l|}{ Frequency } \\
\hline & $\underset{\text { Weekly }}{2}$ & Normal & $>2$ Daily & Variable \\
\hline $\begin{array}{l}\text { Hard } \\
\text { Normal } \\
\text { (Semi) liquid } \\
\text { Variable }\end{array}$ & $\begin{array}{c}46(21.9) \\
6(2.9) \\
0 \\
11(5.2)\end{array}$ & $\begin{array}{c}20(9.5) \\
46(21.9) \\
15(7.1) \\
9(4.3)\end{array}$ & $\begin{array}{r}6(2.9) \\
8(3.8) \\
18(8.6) \\
5(2.4)\end{array}$ & $\begin{array}{l}2(1.0) \\
2(1.0) \\
0 \\
7(3.3)\end{array}$ \\
\hline
\end{tabular}

A positive family history for constipation (meaning that at least one first degree relative was known to have constipation) was present in $90(50 \%)$ of the 180 patients in whom this information was available. Likewise, constipation had been present during infancy in 114 of 202 patients $(56 \%)$. The parents of 82 out of 160 children (51\%) recalled that their child had a fear of defecation as a toddler, resulting in withholding and straining.

Defecation problems versus abdominal pain

As most studies on the treatment and outcome of constipation focus on overt defecation problems, we compared patients presenting with abdominal pain as the sole or major problem ( $n$ $=77$ ) with patients presenting with symptoms classically considered consistent with CFC ('constipation', encopresis, diarrhoea, delayed faecal continence, painful defecation, fear of defecation, and rectal bleeding; table $1 ; n=$ 142). The abdominal pain group tended to be older at the onset of symptoms (mean $85 v 34$ months; $\mathrm{p}<0.001)$; they had a shorter patient's delay (mean $6 v 15$ months; $\mathrm{p}=0.013$ ) and a longer doctor's delay (mean $14 v 5$ months, $\mathrm{p}=0.005)$, resulting in similar total delays (20 $v 19$ months; NS).

TREATMENT

Dietary advice, that is, starting solid foods in infants or introducing fibre rich diets in older children, was the only treatment for 12 patients
(5\%). The other 232 children received lactulose during the evacuation phase at a mean (SD) dose of $0.88(0.46) \mathrm{g} / \mathrm{kg}$ body weight/day (range $0.17-3.45 \mathrm{~g} / \mathrm{kg}$ ). In addition, 183 older children were treated with bisacodyl at a dose of $0.35(0.16) \mathrm{mg} / \mathrm{kg} /$ day (range $0.10-1.07$ $\mathrm{mg} / \mathrm{kg}$ ) and 39 infants and younger children with sodium picosulphate at a dose of 0.23 $(0.11) \mathrm{mg} / \mathrm{kg} /$ day (range $0.06-0.58 \mathrm{mg} / \mathrm{kg}$ ). Mineral oil was prescribed to 53 children at a dose of $1.4(1.0) \mathrm{ml} / \mathrm{kg} /$ day (range $0.2-6.4$ $\mathrm{ml} / \mathrm{kg}$ ). All dosages were taken close to eight weeks after the start of treatment; this is the time expected that the optimal evacuation phase dose was obtained. At some time during the course of treatment it was felt necessary to consult a child psychologist in 57 patients $(23 \%)$, and 31 patients (13\%) were hospitalised at least once for extensive clinical laxation. As a group, patients requiring psychological counselling were not worse than those in whom this was not judged necessary. Ninety five children $(39 \%)$ had a relapse of symptoms, requiring intensification of treatment, at some time before discharge.

At the time this study was performed 212 patients $(87 \%)$ had been discharged from outpatient follow up. The average treatment period had been 13.3 months (median 9 months, range 6 weeks to 6.8 years). One hundred and sixty four (69\%) still used low doses of laxatives at discharge, but in 131 children (62\%) symptoms had disappeared completely at the time of discharge. Improvement of symptoms was obtained in another 70 children (33\%) who had been referred to their general practitioner or were judged able to manage their symptoms themselves. The remaining 11 children (5\%) had unchanged symptoms when last seen at the outpatient department before they discontinued treatment. Thirty two patients were still under supervision. The mean treatment period in this group was longer than average at 23 months, with half of the patients being treated for at least 16 months. No association could be demonstrated between the duration of the treatment period and treatment delay.

\section{Defecation problems versus abdominal pain}

The doses of lactulose and bisacodyl given to patients with abdominal pain were lower than those given to patients with defecation problems: $0.69(0.29) \quad v \quad 0.99 \quad(0.51) \quad \mathrm{g} / \mathrm{kg} /$ day $(\mathrm{p}<0.001)$ and 0.30 (13) $v 0.37 \quad(0.18)$ $\mathrm{mg} / \mathrm{kg} /$ day $(\mathrm{p}=0.006)$, respectively. No significant differences were found for mineral oil and sodium picosulphate, the latter being given to only three children with abdominal pain. The percentages of children requiring psychological consultation or hospital admission were similar in both groups. The mean total duration of treatment in those children discharged from follow up was close to 14 months in both groups. None of the other parameters studied differed significantly between these groups, apart from the presence of encopresis at the time of discharge, which was less often present in the abdominal pain group (5/69) than in the defecation problems group (43/100) 
$(p<0.05)$. The latter was due to the persistence of encopresis in 20 out of 26 patients presenting with encopresis as the main symptom.

FOLLOW UP

Of the 184 patients who were discharged for more than one year we retraced 137 (74\%). These 137 patients were not significantly different in terms of age and symptoms at presentation as compared with the 47 patients not followed up. The median follow up period was four years with a maximum of 13 years. At the time of the interview 90 patients $(66 \%)$ were considered cured, that is, no complaints attributable to CFC and no laxative use during the last six months. Forty seven patients (34\%) still were symptomatic, 12 of them having had complaints ever since discharge. Of the patients who were still experiencing symptoms, only 17 still used laxatives. In all, 54 children (39\%) had experienced a recurrence at some time between discharge and the follow up interview.

For analysis of the data the children were divided into three groups according to their ages at the start of treatment. The persistence of complaints in each group was analysed by survival analysis and Kaplan-Meier profiles were constructed (fig 2). In the group of children who started treatment at ages 13 to 72 months, symptoms persisted for longer periods than they did in the younger and older groups (Mantel-Cox, $\mathrm{p}=0.029$ ). Three years after the start of treatment between 30 and $50 \%$ of the patients were still symptomatic (fig 2).

No association could be demonstrated between outcome on the one hand, and a positive family history of CFC, fear of defecation as a toddler, soiling at the time of presentation, or the need for psychological support during treatment on the other hand. Children who had a history of constipation in infancy tended to do worse at the time of the follow up interview, but this did not reach a significant level. The abdominal pain group performed similarly to the patients presenting with defecation problems.

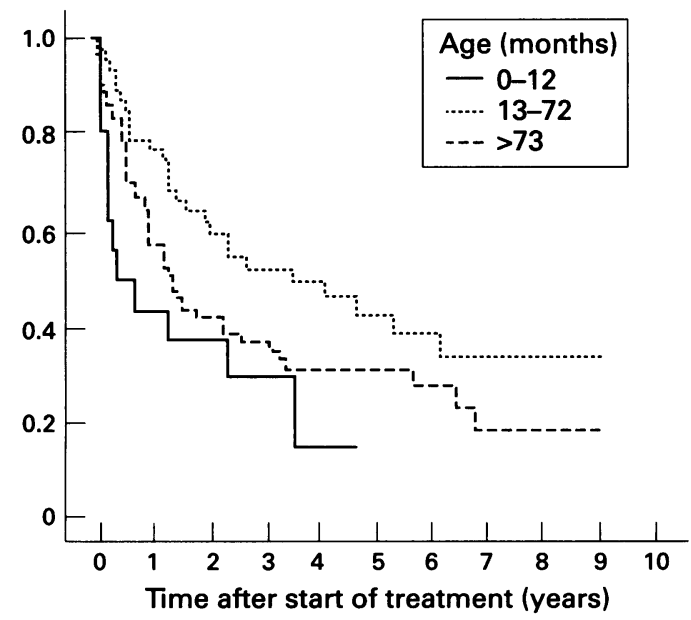

Figure 2 Kaplan-Meier profiles of the number of patients with persisting symptoms of CFC at any time after start of treatment after referral. The group is divided into infants ( $0-12$ months), toddlers (13-72 months), and schoolchildren (> 72 months) according to age at referral.

\section{Discussion}

Loening-Baucke evaluated 174 patients with constipation, all younger than 4 years of age, for symptoms at presentation. ${ }^{5}$ She found infrequent bowel movements in $58 \%$, painful bowel movements in $77 \%$, and stool withholding in $97 \% .^{5}$ In her study, CFC was diagnosed using clinical features only. In our experience, when using clinical features only the diagnosis of CFC may be easily missed. We therefore adopted the Barr score of plain abdominal radiographs as an objective measure of CFC. ${ }^{6}$ In our hands and others', ${ }^{7}$ this method, developed for schoolchildren, is a valuable tool for children over 1 year of age, although (re)validation is impossible for ethical reasons. In recent years new objective criteria have been developed for the assessment of constipation, including the segmental colonic transit time using markers and repeated radiography. ${ }^{8}$ While the use of such a technique would be useful in a prospective study, for clinical purposes the Barr score has the great advantage of enabling an instant diagnosis of constipation and the start of treatment after the first outpatient visit. Although there seems to be no direct relationship between Barr score and colonic transit time, ${ }^{9}$ the same holds for ease of rectal evacuation and colonic transit time in adult constipated patients. ${ }^{10}$ Nevertheless, there is little doubt that increased faecal mass on plain abdominal radiography represents faecal retention and therefore is a simple tool for the objective assessment of constipation. ${ }^{711}$

In the present study, the records of 244 children, aged 0-18 years, with CFC as identified by plain abdominal radiography, were analysed retrospectively. By using this technique we could demonstrate that the clinical picture of CFC is much more diverse than usually acknowledged. Only 46 children (22\%) had the classical presentation of infrequent defecation of increased consistency. Many children had an apparently normal defecation pattern; this is a feature that is not generally appreciated as being consistent with CFC. ${ }^{12}$ The diagnosis may therefore easily be missed, as is illustrated by the long treatment delays, especially in children with abdominal pain as the presenting complaint; this also may explain the large number of patients with occult constipation in our population. Abdominal pain is the most frequently encountered single symptom, occurring in half of our patients. As is clear from the analysis of the abdominal pain group, they essentially differ only from the children presenting with defecation problems in that they are significantly older at the onset of symptoms. The lower doses of laxatives used to reach optimal treatment effect may be fully explained by the higher age at the start of treatment. It is our clinical impression that so-called recurrent abdominal pain in schoolchildren often coexists with CFC. We feel that CFC deserves a prominent place in the differential diagnosis of childhood recurrent abdominal pain..$^{11}{ }^{13}$ In this respect the shorter patient's delay and longer doctor's delay we found are very interesting; they may be interpreted as abdominal pain being a symp- 
tom that provokes more concern in the parents while the doctor fails to make a timely diagnosis of CFC.

To our knowledge, headache and low grade fever have not been reported before as symptoms of CFC. We had several children presenting with temperatures up to $38^{\circ} \mathrm{C}$, or even higher, as one of the main symptoms, CFC being the sole recognised cause. Once the constipation is treated adequately, the body temperature normalises. We are not aware of a report on this phenomenon, nor do we have a sound explanation for it. The importance of it lies in the fact that, apart from simple screening procedures, such as erythrocyte sedimentation rate and white cell count, extensive investigations may be postponed until the effect of laxative treatment is appreciated. In comparison with other studies we found a low prevalence of recurring urinary tract infections: $3.7 \%$ compared with 7-30\%. ${ }^{515-18}$ This may be due to either the high number of cases with occult constipation or to the referral pattern of our patients, half of them being referred to us by general paediatricians. From the literature is known that recurrent urinary tract infections often subside when constipation is adequately treated. ${ }^{19}$

The aetiology of CFC is still unknown. Some children demonstrate unconscious contractions of the external anal sphincter during defecation. ${ }^{20}$ This paradoxical reaction is probably based on fear of a painful defecation and may contribute to the pathogenesis of CFC because it limits colonic evacuation. Familial and constitutional factors seem to play a part in the aetiology of CFC. In half of our patients the parents reported that they themselves or one of the other children also had constipation, which is in agreement with other reports. ${ }^{16} 182122$ Both the positive family history and the fact that $57 \%$ of our patients were reported to have suffered from constipation as an infant suggests a role for congenital factors in the aetiology of CFC.

Although in $23 \%$ of the families in our study the help of a child psychologist was sought, this was mostly the case only after our regular treatment regimen failed to reach any further improvement, or when overt psychosocial problems seemed to interfere. It was thought by us and by the team of psychologists that the behavioural problems were not the cause of the defecation abnormalities in any of the patients. These problems often seem to be a sequel of CFC and hence decrease after successful treatment. ${ }^{161821-24}$

Contrary to our experience that encopresis is virtually always accompanied by CFC, Benninga et al recently suggested that encopresis could exist without CFC. ${ }^{25}$ In their study, constipation was primarily diagnosed on clinical grounds. Defecation dynamics as well as child behaviour scores were found abnormal in children with CFC with or without encopresis, and in children with encopresis without CFC, compared with controls. ${ }^{25}$ Rockney et al, using plain abdominal radiographs for diagnosis, found about one fifth of the encopretic children to have no evidence of faecal reten- tion. ${ }^{7}$ These children were less likely to have faeces palpable on rectal examination, and more likely to have experienced difficulties in toilet training.

Recently, Loening-Baucke published a study of CFC in children younger than 4 years of age. ${ }^{5}$ The treatment regimen in this study was similar to ours, although we do not routinely use enemas and never prescribe milk of magnesia. We conclude with her that the laxative doses required to adequately treat constipation in children is much higher than the doses suggested by the manufacturer. The results of our treatment strategy are comparable with hers ${ }^{5}$ and to those of other studies. ${ }^{2} 4182627$

From our follow up data it may be concluded that for the majority of children, although symptoms from CFC may be tenacious, the long term prognosis is favourable. According to our results, children starting treatment before 13 months of age had a better prognosis than those starting treatment between 13 and 72 months of age. This may be due to difficulties associated with bowel habit training, as this is the age that continence must be accomplished. For those children that have persistent symptoms of constipation, biofeedback training might be a valuable alternative to conventional treatment. ${ }^{28-30}$

In summary, CFC, which may be difficult to recognise when classical symptoms of disordered defecation are absent, can effectively be controlled in the majority of children when using an extensive oral laxation regimen. As a rule, long term prognosis is favourable, independent of the presenting symptoms.

1 Loening-Baucke VA, Cruikshank BM. Abnormal defecation dynamics in chronically constipated children with encopreis. F Pediatr 1986; 108: 562-6.

2 Levine $M D$, Bakow $H$. Children with encopresis: a study of treatment and outcome. Pediatrics 1976; 58: 845-52.

3 Molnar D, Taitz LS, Urwin OM, et al. Anorectal manometry results in defecation disorders. Arch Dis Child 1983; 58 257-61.

4 Taitz LS, Wales JKH, Urwin OM, et al. Factors associated with outcome in management of defecation disorders. Arch Dis Child 1986; 61: 472-7.

5 Loening-Baucke V. Constipation in childhood: patient characteristics, treatment, and long-term follow-up. Gut 1993; 34: 1400-4.

6 Barr RG, Levine MD, Wilkinson RH, et al. Chronic and occult stool retention. A clinical tool for its evaluation in school-aged children. Clin Pediatr (Phila) 1979; 18: 674-9.

7 Rockney MR, McQuade WH, Days AL. The plain abdominal roentgenogram in the management of encopresis. Arch Pediatr Adolesc Med 1995; 149: 623-7.

8 Metcalf AM, Phillips SF, Zinsmeister AR, MacCarty RL, Beart RW, Wolff BG. Simplified assessment of segmental colonic transit. Gastroenterology 1987; 92: 40-7.

9 Benninga MA, Büller HA, Staalman CR, et al. Defecation disorders in children, colonic transit time versus the Barrscore. Eur $¥$ Pediatr 1995; 154: 277-84.

10 Karlbom U, Påhlman L, Nilsson S, Graf W. Relationship between defecographic findings, rectal emptying, and colonic transit time in constipated patients. Gut 1995; 36: 907-12.

11 Fenton TR. The irritable bowel syndrome. A paediatric entity? In: Milla PJ, ed. Disorders of gastrointestinal motility in childhood. Chichester: John Wiley, 1988:101-9.

12 Cucchiaria S, Coremans G, Staiano A, et al. Gastrointestinal transit time and anorectal manometry in children with transit time and anorectal manometry in children with
fecal soiling. $\mathcal{F}$ Pediatr Gastroenterol Nutr 1984; 3: 545-50.

fecal soiling. F Pediatr Gastroenterol Nutr 1984; 3: 545-50.
13 Dimson SB. Transit time related to clinical findings in children with recurrent abdominal pain. Pediatrics 1971; 47: dren with

14 Stone RT, Barbero GJ. Recurrent abdominal pain in childhood. Pediatrics 1970; 45: 732-8.

15 Loening-Baucke VA. Factors determining outcome in children with chronic constipation and faecal soiling. Gut 1989; 30: 999-1006.

16 Clayden GS, Lawson JON. Investigation and management of long-standing chronic constipation in childhood. Arch Dis Child 1976; 51: 918-23. 
17 Levine MD. Children with encopresis: a descriptive analysis. Pediatrics 1975; 56: 412-6.

18 Abrahamian JP, Lloyd-Still JD. Chronic constipation in childhood: a longitudinal study of 186 patients. $\mathcal{f}$ Pediatr Gastroenterol Nutr 1984; 3: 460-7.

19 O'Regan S, Yazbeck S, Hamberger B, et al. Constipation a commonly unrecognized cause of enuresis. Am $\mathcal{F}$ Dis Child 1986: 140: 260-1

20 Loening-Baucke VA. Modulation of abnormal defecation dynamics by biofeedback treatment in chronically constipated children with encopresis. $f$ Pediatr 1990; 116: 214-22.

21 Lowery SP, Srour JW, Whitehead WE, et al. Habit training as treatment of encopresis secondary to chronic constipation. F Pediatr Gastroenterol Nutr 1985; 4: 397-401.

22 Davidson M, Kugler MM, Bauer CH. Diagnosis and mancted constipation and obstipation. $\mathcal{F}$ Pediatr 1963; 62: 261-75.

23 Hatch TF. Encopresis and constipation in children. Pediatr Clin North Am 1988; 35: 257-80.

24 Younoszai MK, Tolaymat N. Chronic functional constipaYounoszai MK, Tolaymat N. Chronic functional constipa-
tion in infants and children. In: Lebenthal E, ed. Textbook of gastroenterology and nutrition in infancy. New York: Raven Press, 1989:1311-26.

25 Benninga MA, Büller HA, Heymans HSA, et al. Is encopresis always the result of constipation? Arch Dis Child 1994; 71: 186-93.

26 Nolan T, Debelle G, Oberklaid F, et al. Randomised trial of laxatives in treatment of childhood encopresis. Lancet 1991; 338: 523-7.

27 Loening-Baucke VA, Younoszai MK. Abnormal ana sphincter response in chronically constipated children. $\mathscr{f}$ Pediatr 1982; 100: 213-8.

28 Olness K, McParland FA, Piper J. Biofeedback: a new modality in the management of children with fecal soiling. I Pediatr 1980; 96: 505-9.

29 Whitehead WE. Treatment of fecal incontinence in children with spina bifida: a comparison of biofeedback and behaviour modification. Arch Phys Med Rehabil 1986; 67: 218-24.

30 Benninga MA, Büller HA, Taminiau JAJM. Biofeedback training in chronic constipation. Arch Dis Child 1993; 68 126-9. 